\title{
Lettre d'Andreï Pachkov sur la mort d'Alexandre Pouchkine, datée du 30 janvier 1837 (archives du prince Valentin Chakhovskoii)
}

\section{Galina Sedova}

Traducteur : Dmitry Kudryashov

\section{(2) OpenEdition}

Journals

Édition électronique

URL : https://journals.openedition.org/rbnu/3139

DOI : 10.4000/rbnu.3139

ISSN : 2679-6104

\section{Éditeur}

Bibliothèque nationale et universitaire de Strasbourg

\section{Édition imprimée}

Date de publication : 1 mai 2010

Pagination : 62-65

ISSN : 2109-2761

\section{Référence électronique}

Galina Sedova, « Lettre d'Andreï Pachkov sur la mort d'Alexandre Pouchkine, datée du 30 janvier 1837 (archives du prince Valentin Chakhovskoï) », La Revue de la BNU [En ligne], 1 | 2010, mis en ligne le 01 janvier 2021, consulté le 12 juin 2021. URL : http://journals.openedition.org/rbnu/3139 ; DOI : https:// doi.org/10.4000/rbnu.3139

La Revue de la BNU est mise à disposition selon les termes de la Licence Creative Commons Attribution - Pas d'Utilisation Commerciale - Partage dans les Mêmes Conditions 4.0 International. 


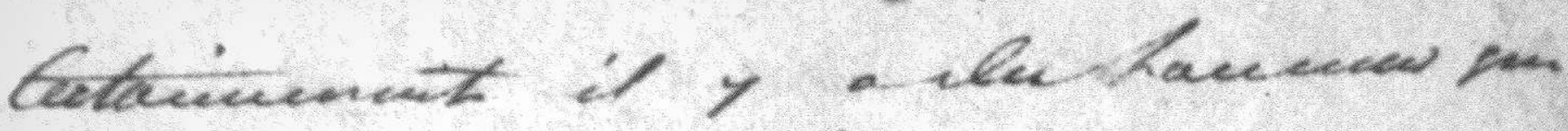

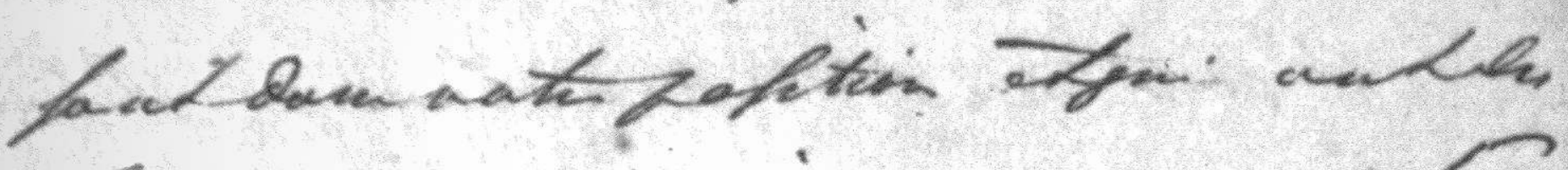

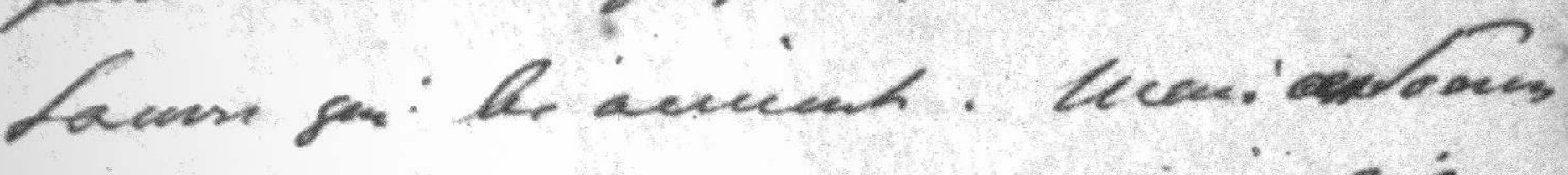

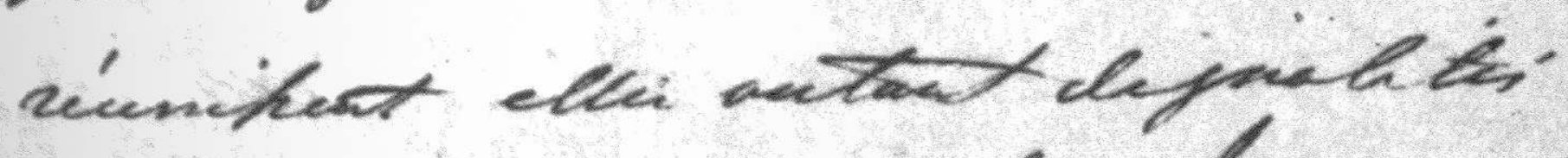

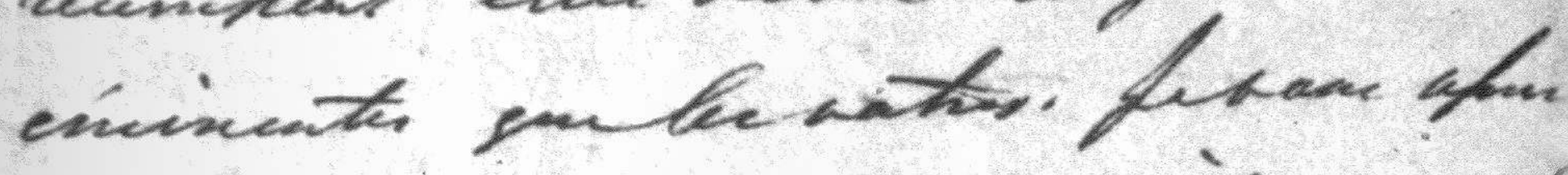

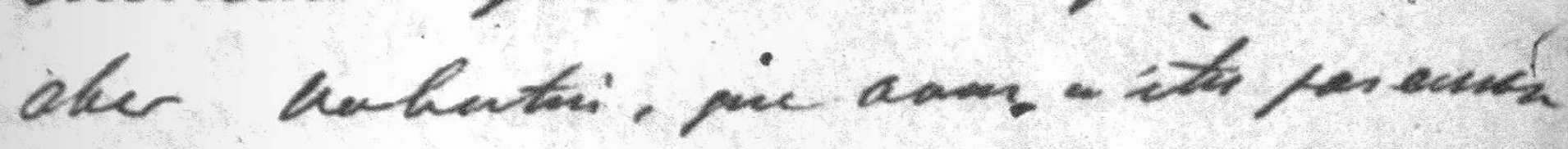
tod at fort unelewened.-Couparen

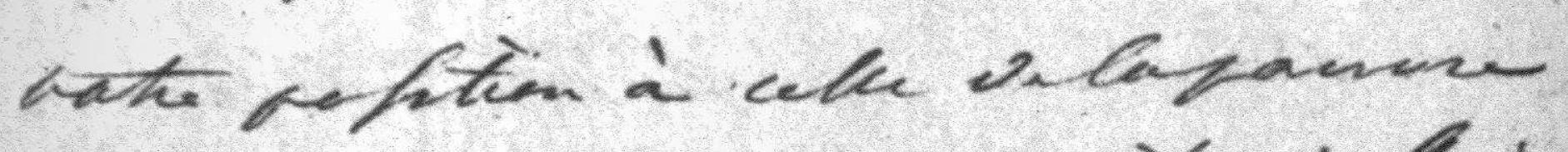

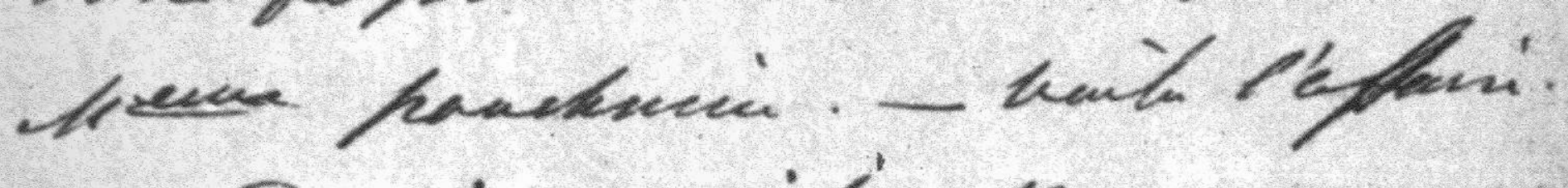
for. Pantai, a prifit Heckeren.

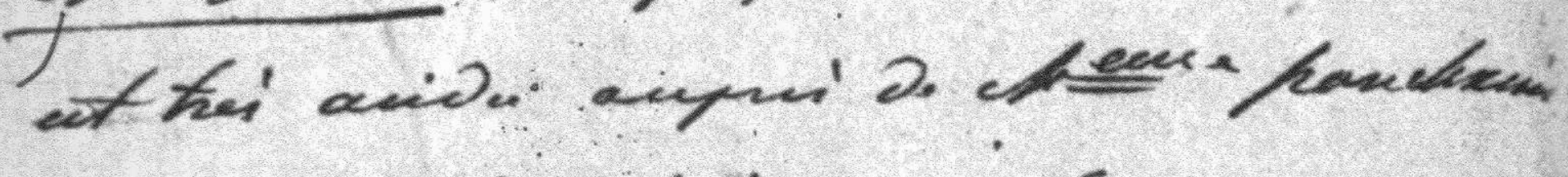

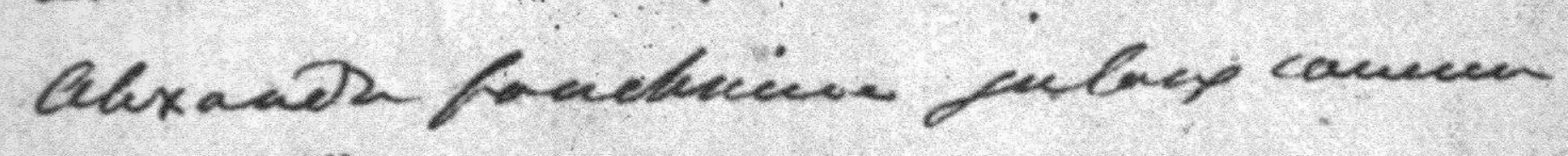

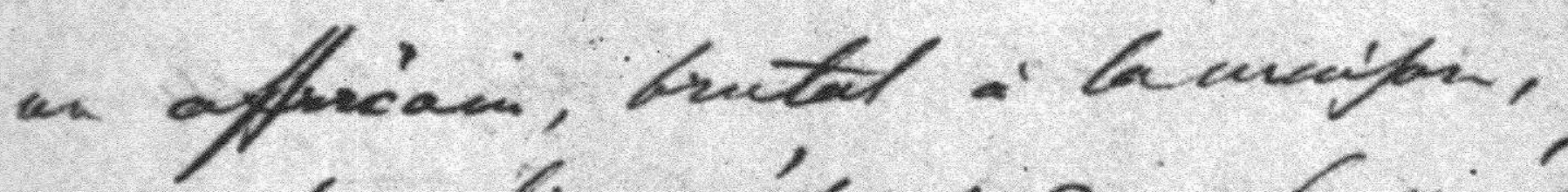

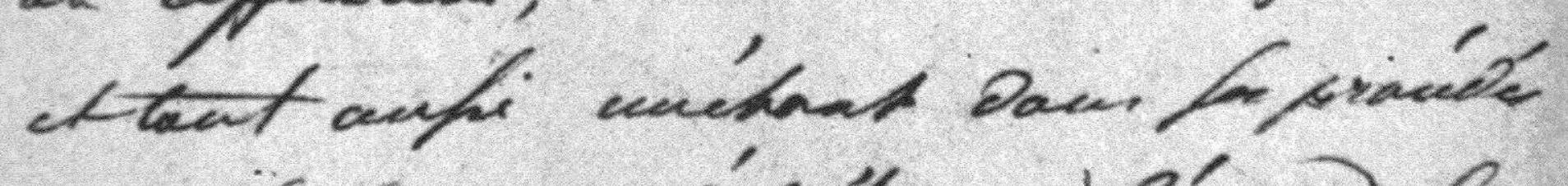

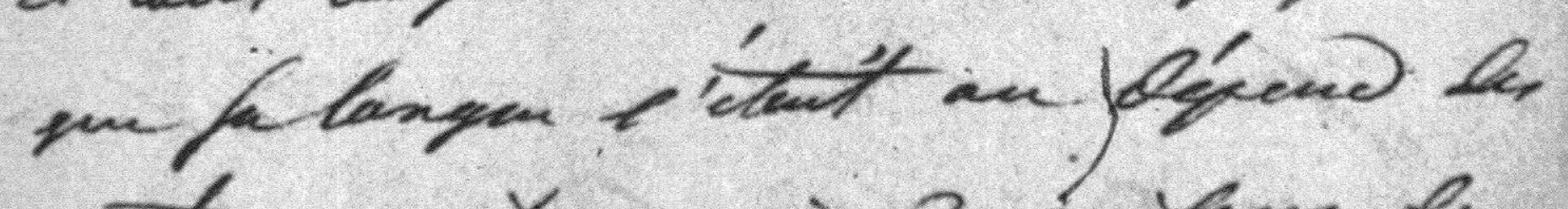

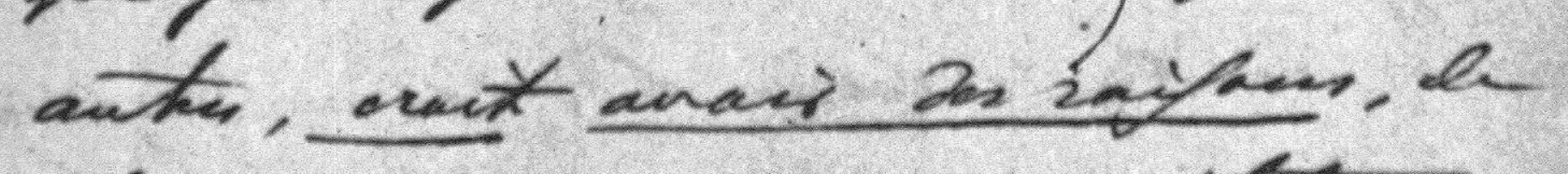

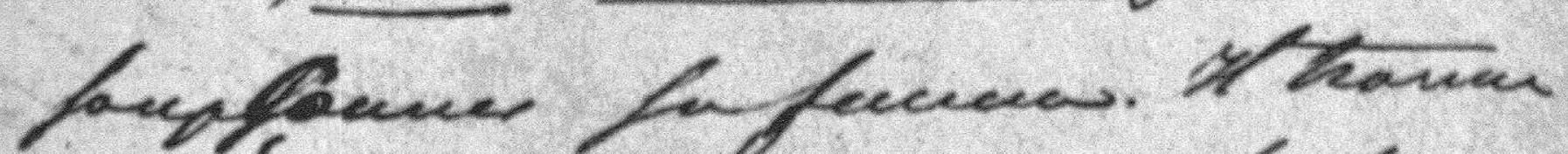




\title{
Lettre d'Andreï Pachkov
}

\section{sur la mort d'Alexandre Pouchkine}

\author{
datée du 30 janvier I 837 (archives du prince Valentin Chakhovskoï)
}

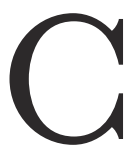

ette lettre est conservée dans les archives familiales du prince Valentin Mikhaïlovitch Chakhovskoï (1801-1850). Après 1917, ces archives ont été enlevées du domaine familial (à Belaya Kolp, dans le district de Volokolamsk, lui-même situé dans la province de Moscou) et nationalisées. Elles sont actuellement conservées au Département des manuscrits de la Bibliothèque d'Etat de Russie, à Moscou, qui a bien voulu fournir une copie numérisée de la lettre afin de pouvoir la publier ici. Ce document présente un intérêt certain pour les historiens de la littérature russe, dans la mesure où il a été écrit le lendemain de la mort de Pouchkine et contient nombre de données jusqu'ici inconnues sur les événements qui ont conduit au duel fatal du poète avec l'officier du régiment de cavalerie Georges d'Anthès.

L'auteur de la lettre, le général en retraite Andreï Ivanovitch Pachkov (1792-1850) connaissait personnellement Pouchkine et sa femme et les a plusieurs fois rencontrés dans la haute société de SaintPétersbourg. La femme de Pachkov, Adélaïde Modene (1803-1844) faisait partie, elle aussi, des amis proches du poète. Elle était la fille d'un émigré français, le comte Gabriel Carlovitch de Raymond-Modene (17741833), qui s'est mis au service de la Russie en 1798 au poste de " Jägermeister-chef ", et d'Elisabeth Nikolaevna Saltykova (1773-1852).

De par ses relations familiales et mondaines, l'auteur de la lettre a pu discuter de l'histoire du duel de
Pouchkine et donner son avis sur les circonstances qui ont conduit à cette tragédie. Exprimant l'opinion largement répandue dans la haute société, Pachkov révèle dans cette lettre que le conflit entre Pouchkine et d'Anthès était le résultat de plusieurs composantes : notamment de la négligence de l'épouse du poète, qui acceptait la galanterie persistante de d'Anthès. Mais Pachkov note également que le comportement du jeune homme vis-à-vis d'une femme mariée était très grave. L'histoire racontée dans cette lettre était jusqu'alors inconnue dans les études pouchkiniennes ; en tout cas, selon les règles de l'étiquette, il était impossible durant un bal d'offrir à une femme une côtelette... Pachkov attire également l'attention sur le fait que la réaction négative de Pouchkine face au comportement du jeune homme avait été renforcée, et même en partie déclenchée par l'attitude des connaissances mondaines du poète, comme si elles s'étaient donné pour objectif de l'agacer de toutes les façons et de le pousser au conflit avec d'Anthès. Pachkov relaie ici l'opinion de nombreux cancaniers mondains, expliquant le caractère émotif du poète par son ascendance africaine (l'arrière grand-père maternel de Pouchkine était originaire de ce continent). Le narrateur, comme beaucoup de ses contemporains, manquait d'informations sur les causes véritables de la tragédie du poète ; il était convaincu que la responsabilité de ce qui lui était arrivé lui incombait en grande partie à lui-même. Tout en rendant hommage à son esprit, il pointe ce qui lui semble être une absence de bon sens. 


\section{"Heeckeren a l'imprudence à une soirée d'aller faire un tour de valse avec Mme Pouchkine et à souper d'aller lui porter une côtelette"}

Comme beaucoup de gens de son entourage, Pachkov ne pensait pas qu'il soit nécessaire de parler de l'importance de la perte de Pouchkine pour la culture russe. Se référant à cette tragédie, il demande simplement à son ami de comparer sa propre situation de veuf avec celle de " la pauvre Mme Pouchkine ", présentée dans la lettre non pas comme la veuve d'un grand poète, mais comme une femme du monde qui a perdu son mari et, peut-être, sa réputation, et dont le sort est semblable à celui du destinataire de la lettre, avec dans les deux cas de petits enfants à leur charge.

La lettre publiée ici attire l'attention du lecteur d'aujourd'hui sur cette partie de la société métropolitaine qui n'était nullement hostile à l'homme Pouchkine, mais qui est restée totalement indifférente au poète, pendant sa vie comme après sa mort, et qui considérait l'incident comme une histoire mondaine parmi d'autres. Ce n'est pas un hasard si l'histoire de Pouchkine est encadrée dans la lettre par des informations sur les affaires d'argent de l'auteur (litiges avec sa mère pour l'héritage) et sur la vie personnelle du destinataire (les enfants Chakhovskoï et ses sœurs).

La lettre est écrite en français à l'encre dite " de noix de galle " (encre brune utilisée pendant plusieurs siècles en Russie), qui a disparu par endroits. La difficulté principale dans le déchiffrement réside dans l'utilisation d'une écriture cursive et parfois d'un certain laisser-aller dans la formation des lettres, le placement des accents, l'utilisation des virgules. Il semble que, comme la plupart des aristocrates russes, l'auteur de la lettre ait été depuis l'enfance familier du français parlé et que c'est cette langue parlée qu'il avait l'habitude de coucher sur le papier.

La transcription respecte fidèlement l'original, y compris dans ses maladresses ou ses approximations de langue. Seules la ponctuation et l'orthographe, quand le maintien de l'original aurait été gênant pour une bonne compréhension du texte, ont été rétablies conformément au bon usage d'aujourd'hui.

\section{Galina Sedova \\ (traduction Dmitry Kudryashov)}




\section{Reçu le 3 février 1837 30 janvier I837. Petersbourg}

Bien cher Valentin, je viens de recevoir votre lettre du 26 en réponse à la mienne. Je ne suis pas aussi coupable que vous le pensez. Car il me semble que je dois avoir dit dans ma lettre que j'envoie la copie du papier expédié à la direction régionale, à Khokhlov pour vous la présenter. Aurais-je oublié de vous le dire ? Dans tous les cas le papier a été envoyé à Khokhlov : en attendant s'il pouvait être arrivé qu'il ne l'ait pas reçu, je vous en envoie ci joint un duplicatum.

Ces Messieurs sont bien étranges de ne vouloir pas délivrer un papier, le seul même qu'ils sont en devoir de me donner, car c'est la principale raison qui m'a fait désirer d'avoir un arbitrage. Car si ce n'était ma mère, je m'en serais parfaitement passé et je vous assure que je n'y aurais pas perdu : enfin puisque vous avez eu la complaisance de leur demander à voir leur tâtonnement en affaire, j'attendrai le résultat de votre décision, étant persuadé du coup d'œil juste que vous avez en général sur les affaires. Jusque-là je patiente.

Mon cher ami, votre position quoique bien cruelle a du charme encore. Voyez autour de vous ; que de chers et d'excellents êtres qui vous entourent, qui ne vivent que pour vous et par vous. Rendez grâces au Tout-puissant. On n'est pas encore le plus malheureux quand on a trois enfants et des sœurs comme vous en avez. Ce sont des amies si sincèrement dévouées, qui ont fait abnégation d'elles-mêmes pour vous, que vous êtes encore à mes yeux un être privilégié. Certainement il y a des hommes qui sont dans votre position et qui ont des sœurs qui les aiment. Mais ces sœurs réunissent-elles autant de qualités éminentes que les vôtres ? Je vous assure, cher Valentin, que vous n'êtes pas encore tout à fait malheureux. Comparez votre position à celle de la pauvre Mme Pouchkine. Voilà l'affaire. Mr Dantès, à présent Heeckeren, est très assidu auprès de Mme Pouchkine. Alexandre Pouchkine jaloux comme un Affricain, brutal à la maison, et tout aussi méchant dans ses procédés que sa langue l'était au dépend des autres, croit avoir des raisons de soupçonner sa femme. Il trouve naguère Mr Dantès chez sa femme, dans un moment où il ne l'y attendait pas. Il lui fait des reproches. Je dois ici dire qu'en général, on dit qu'il n'y avait qu'imprudence de la part de Mme Pouchkine. J'arrive à le croire, car premièrement, que l'on a toujours les temps de croire le mauvais côté. Et secondement, qu'il est fort facile aux hommes de perdre une femme sans qu'elle l'ait le moins mérité. Revenons au fait. Heeckeren, pour lui-même, ou pour Mme Pouchkine prétend que M. Pouchkine le soupçonne injustement, car il n'était venu que pour demander la main de sa belle-sœur. Or l'affaire s'arrange, le mariage a lieu. Tout était donc fini. L'honneur de la femme mis à couvert. Mais cependant comme le monde est méchant il s'est trouvé des gens qui ont fait l'infâme métier de les jeter l'un contre l'autre.

On est venu dire à Pouchkine et on lui a envoyé des lettres anonymes dans lesquelles on le prévenait que Heeckeren avait dit telle et telle chose sur lui et sa femme. A Heeckeren on venait dire que Pouchkine l'avait cherché à telle ou telle soirée pour lui donner un soufflet en présence de tout le monde. Enfin Heeckeren a l'imprudence à une soirée d'aller faire un tour de valse avec Mme Pouchkine et à souper d'aller lui porter une côtelette. Voilà que Pouchkine écrit une lettre à Heeckeren père, si hors de mesure et si forte, qu'il a été impossible de ne pas en venir à un duel. Ils se sont battus à 6 pas. Heeckeren a tiré le premier. Il a tiré juste.

La balle est entrée dans l'estomac et est restée dans le corps. Pouchkine est tombé. Mais il a exigé que Heeckeren reste à sa place et couché, il a tiré, et l'a blessé au bras.

Enfin, Pouchkine ayant ainsi perdu sa femme, est mort hier à trois heures après midi.

Voyez je vous prie la position de sa pauvre femme avec 4 ou 5 enfants, perdue de réputation et dans quelle malheureuse relation vis-à-vis de sa sœur. Car à présent, à qui peut-on persuader qu'il n'y ait pas eu des raisons

bien fortes, pour que deux beaux-frères se battent à mort ? On prétend qu'il est mort en disant que s'il en réchappait, il recommencerait encore et ne serait tranquille que quand il aurait tué Heeckeren. Il n'est question que de cette malheureuse histoire. Toutes les dames en ont les nerfs dérangés. Les uns donnent tort à la femme,

les autres à Pouchkine, les troisièmes à Heeckeren. Moi, je plains la pauvre femme, qui peut-être pour une imprudence est à jamais perdue. Pouchkine avec tout son esprit n'a jamais eu un brin de bon sens. Mais en voilà assez sur ce sujet.

Que Dieu vous conserve en bonne santé, vous et tous les vôtres. Embrassez vos chers enfants, parlez-leur de nous, comme de vos vrais et dévoués amis, afin qu'ils nous connaissent depuis leurs jeunes années. Mes tendres amitiés à vos bonnes sœurs. J'ai écrit à Cléopâtre depuis que je vous ai envoyé ma dernière lettre, je pense que j'aurai le bonheur de recevoir une réponse d'elle. Que Dieu veille sur cet être angélique! Adieu, cher et excellent ami. Adèle réunit tous ses vœux aux miens pour tout ce qui vous regarde tous. 